\title{
Suppression of Natural Convection in a Thermoacoustic Pulse Tube Refrigerator *
}

\author{
HAN Jun-Qing(韩君庆), LIU Qiu-Sheng(刘秋生)** \\ Key Laboratory of Microgravity, Institute of Mechanics, Chinese Academy of Sciences, Beijing 100190
}

(Received 5 November 2012)

\begin{abstract}
The effects of gravity on the efficiency of thermoacoustic engines are investigated theoretically and experimentally, especially for thermoacoustic pulse tube refrigerators. The significant effects of gravity are found to be due to the presence of natural convection in the thermoacoustic pulse tube when the hot side of the tube is lower than the cold side. This kind of natural convection influences and reduces the efficiency of the thermoacoustic working system. Thus, how to suppress this natural convection becomes important for increasing the efficiency of thermoacoustic engines. Unlike the method of inserting a silk screen in a pulse tube, the present study uses a numerical simulation method to research the natural convection in pulse tubes, and we try to change the shape of the pulse tube to suppress this convection.
\end{abstract}

PACS: 43.35.Ud, 44.25.+f, 44.05.+e $\quad$ DOI: $10.1088 / 0256-307 \mathrm{X} / 30 / 5 / 054301$

Natural convection in cavities has received considerable attention from many researchers experimentally and numerically due to the importance of such geometry in solar receiver systems, fire research, electronic cooling, the brake housing of aircraft, and many environmental geothermal processes. ${ }^{[1]}$ Lots of methods are used to boost natural convection, including experiments and theories, for example, natural convection in periodic boundaries. ${ }^{[2,3]}$ However, it is not necessary to strengthen natural convection in all conditions. On the contrary, sometimes we need to suppress it. A very persuasive example is the thermoacoustic engine, where we need to suppress natural convection in the pulse tube, with the purpose of increasing the efficiency of the engine. Gravity properties ${ }^{[4-6]}$ exist in pulse tube refrigerators, and are demonstrated when the gravity affects the efficiency of the thermoacoustic engine when the hot side of the pulse tube is lower than the cold side. Fujimoto et al. ${ }^{[7]}$ reported the gravity orientation dependence for pulse tube refrigerators driven at a low frequency $(\sim 2 \mathrm{~Hz})$. Johnson et al. ${ }^{[8]}$ well characterized the thermal loads associated with free convection within a non-operating cooler. They presented representative data for the angular dependency and found the 'off-state' convective load. In order to quantify the observed orientation effects with high-frequency Stirling-type pulse tube cryocoolers, a series of detailed tests by Ross ${ }^{[6]}$ were conducted to generate performance data on both U-tube and linear type pulse tubes of two different manufacturers as functions of orientation angle and compressor drive level. To suppress natural convection, Thummes et $a l .{ }^{[4]}$ systematically researched the gravity property in a pulse tube refrigerator and introduced the theory of natural convection in order to explain this appearance. They inserted a silk screen in a pulse tube to decrease the effect of gravity. Kasthurirengan et al. ${ }^{[9,14]}$ also adopted a filling silk screen to decrease the gravity effect. Shiraishi et al. ${ }^{[10,11]}$ tried to import additive direct current to suppress the gravity effects. However, these methods are unsatisfactory for suppressing gravity effects.

By working on the mechanism of natural convection, unconsciously we find that the cavity shape only changes from rectangular to taper when the other conditions stay the same, and the natural convection is amazingly suppressed. Then we analyze the reasons why the taper can reduce the natural convection. Next, we apply this idea to thermoacoustic engines to see if the taper pulse tube can also reduce natural convection when we add an oscillating flow. Fortunately, we obtain the same results.

The numerical model for pure natural convection without oscillating flow is shown in Fig. 1. The cavity can rotate in the plane in order to obtain various angles between gravity inclination and temperature gradient. The right and left walls are adiabatic. The cool and hot walls are isothermal. Here, $\alpha$ is the angle between the gravity inclination and the temperature gradient; $\xi$ is the ratio between the cool and hot wall lengths:

$$
\xi=\frac{L_{\text {coolwall }}}{L_{\text {hotwall }}}
$$

When $\xi$ is equal to 1 , the cavity is rectangular. Otherwise, the cavity has a taper. We set the height of enclosure geometry $H=60 \mathrm{~mm}$. Though the lengths of the cool and hot walls change as we set different $\xi$, $L_{\text {coolwall }}+L_{\text {hotwall }}=12 \mathrm{~mm}$ is sustained. The above reference values come from a real thermoacoustic pulse tube refrigerator. ${ }^{[10]}$

In our study, we use ideal air as the work medium in order to obtain more accurate results. The flow is assumed to be laminar. The development of natural convection is governed by the Navier-Stokes equations for compressible fluid. The mass, momentum, energy and gas state equations for the working gas are ex-

\footnotetext{
*Supported by the National Natural Science Foundation of China under Grant Nos 50890182 and 11072249.

** Corresponding author. Email: liu@imech.ac.cn

(C) 2013 Chinese Physical Society and IOP Publishing Ltd
} 
pressed as ${ }^{[15]}$

$$
\begin{aligned}
& \frac{\partial \rho}{\partial t}+\nabla(\rho \boldsymbol{v})=0, \\
& \frac{\partial}{\partial t}(\rho \boldsymbol{v})+\nabla(\rho \boldsymbol{v} \boldsymbol{v})=\boldsymbol{F}+\nabla(\boldsymbol{\tau}), \\
& \frac{\partial}{\partial t}(\rho E)+\nabla(\boldsymbol{v}(\rho E+p))=\nabla(k \nabla T+(\boldsymbol{\tau} \boldsymbol{v})), \\
& p=\rho R T,
\end{aligned}
$$

where $E$ is the total energy, and $E=h-p / \rho+v^{2} / 2$, $\tau$ is the stress tensor, $k$ is the thermal conductivity, $R$ is the gas constant, and $\boldsymbol{F}$ is the body force.

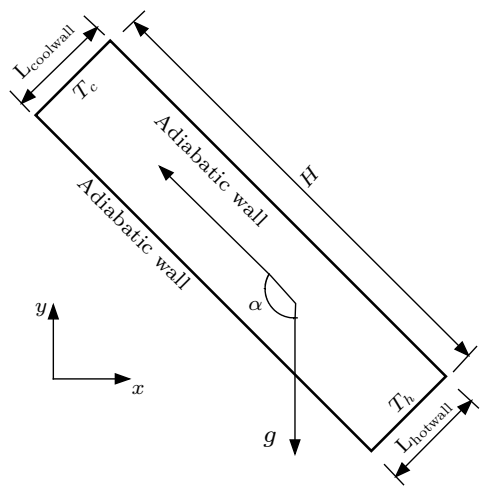

Fig. 1. Enclosure geometry and temperature boundary conditions.

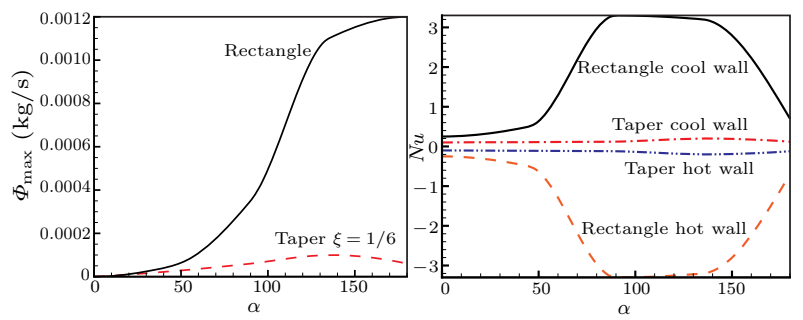

Fig. 2. (a) Maximum streaming function value distribution, and (b) $N u$ for cool and hot walls in different inclination angles $\alpha$ for $\xi=1 / 6$.

Here we define non-dimensional $N u$ in the following, which is similar to the definition in Ref. [13].

$$
N u=-\frac{1}{\Delta T} \int_{0}^{L} \frac{\partial T}{\partial x} d y .
$$

The stream function is formulated as a relation between the streamlines and the statement of the conservation of mass. A streamline is a line that is tangent to the velocity vector of the flowing fluid. For a 2D planar flow, the stream function $\Phi$ is defined as

$$
\rho u=\frac{\partial \Phi}{\partial y} ; \quad \rho v=-\frac{\partial \Phi}{\partial x} .
$$

We define the $R a$ number used to measure the intensity of natural convection,

$$
R a=\frac{g \beta\left(T_{\mathrm{h}}-T_{\mathrm{c}}\right) H^{3}}{\nu \kappa},
$$

where $T_{\mathrm{c}}$ is the temperature of the cool wall, $T_{\mathrm{h}}$ the temperature of the hot wall, $\nu$ the kinematic viscosity coefficient, $\kappa$ the thermal diffusivity, and $\beta$ the expansion coefficient.

We then numerically study the natural convection in different inclination angles by the finite volume method with the SIMPLE algorithm. ${ }^{[12]}$ A boundary layer mesh is used to obtain accurate details of the flow field.

Firstly, we introduce the results in the condition $\xi=1 / 6$, and then show the other $\xi$ results. Here we set the temperature of the hot wall $T=300 \mathrm{~K}$ and the temperature of the cool wall $T=100 \mathrm{~K}$. The temperature difference, $\Delta T$, between the cool and hot walls is $200 \mathrm{~K}$. The referenced parameter values are $g=9.8 \mathrm{~m} / \mathrm{s}^{2}, \beta=1 / 300 \mathrm{~K}, T_{\mathrm{h}}=300 \mathrm{~K}, T_{\mathrm{c}}=100 \mathrm{~K}$, $L=0.06 \mathrm{~m}, H=0.012 \mathrm{~m}, \mu=1.8 \times 10^{-5} \mathrm{~kg} / \mathrm{ms}, \nu=$ $\mu / \rho, k=0.0242 \mathrm{~W} / \mathrm{mK}, \kappa=k / \rho c_{\mathrm{p}}, \rho=1.25 \mathrm{~kg} / \mathrm{m}^{3}$. Thus, when $\Delta T$ is equal to $200 \mathrm{~K}, R a=2 \times 10^{6}$. We will introduce other $\Delta T$ subsequently.

Figure 2(a) shows the maximum value of the streaming function at different inclination $\alpha$. We find that when the taper presents, the value of the streaming function is smaller than the case without taper. Thus, the flow intensity is reduced by the taper and natural convection is suppressed. Figure 2(b) is the $N u$ for cool and hot walls in different inclinations. We find that when there is a taper, $N u$ is obviously smaller than that for the rectangle in the cool and hot walls. The $N u$ number also shows that the natural convection is suppressed by a taper, which is consistent with the analysis in Fig. 2(a).

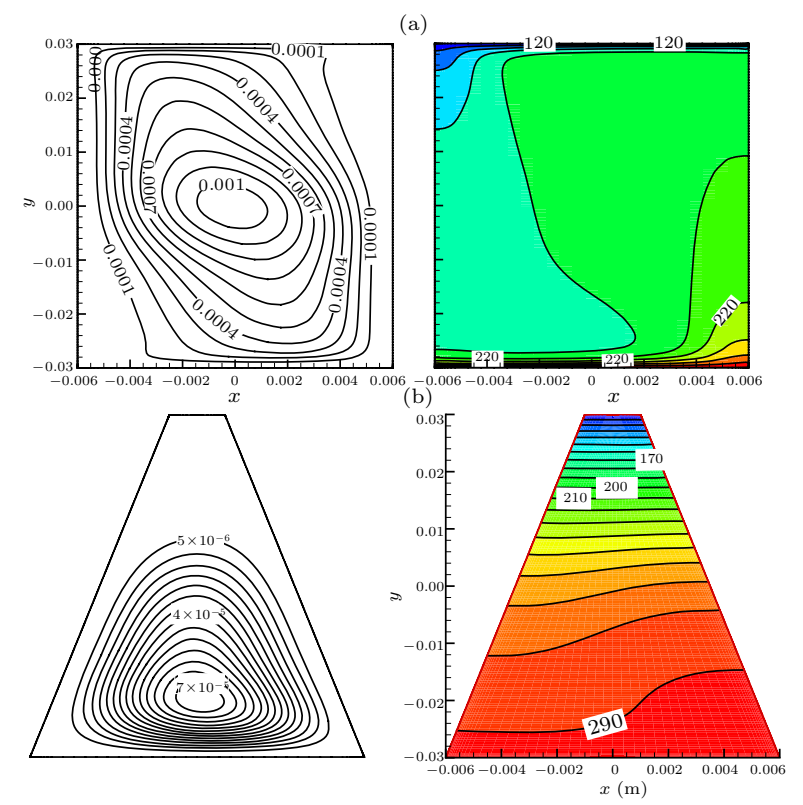

Fig. 3. The structure of the flow field in the rectangular (a) and taper (b) cavities.

We find that when the inclination is about $135^{\circ}$, the natural convection is strongest from the variation of $N u$. Thus, this is a foreshadowing. Next we choose the flow field in this inclination angle to explain why 
natural convection is suppressed when there is a taper. Figures 3(a) and 3(b) are the structures of the flow field, which correspond to rectangular and taper cavities with $\xi=1 / 6$, respectively. From left to right are the stream function and the temperature distribution, in turn, in these two figures. Comparing these two groups of streaming functions in different pulse tube shapes, we find that when there is no taper, the convective cell is filled all over the cavity. The air of all the volume participates in the heat transfer cycle. However, if there is a taper, the streamline covers almost all the hot bottom. In the top zone, the flow is very weak. Convective heat exchange because of air flow is restricted by a taper for the top part of the cavity through the analysis of stream function distribution. Thus, it is implied that a taper suppresses flow intensity.
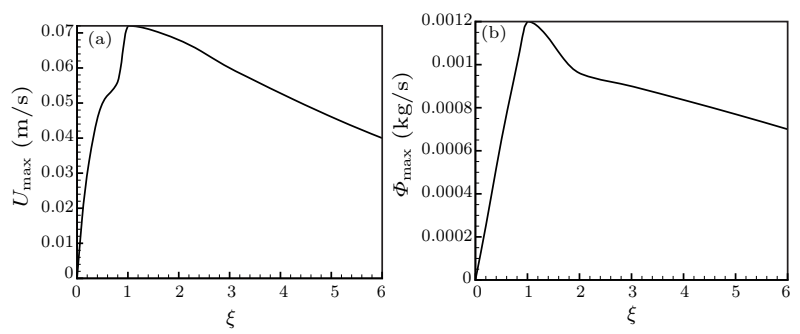

Fig. 4. Maximum velocity and streaming function values in different $\xi$ for inclination $\alpha=180^{\circ}$ : (a) $\max$ velocity value, (b) max streaming function value.
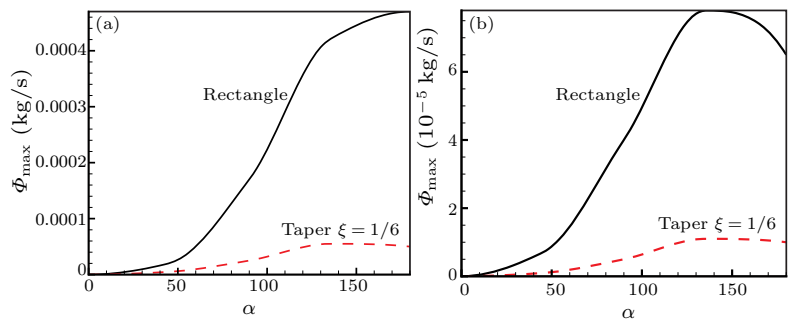

Fig. 5. Maximum streaming function value in different inclination angles $\alpha$ with $\xi=1 / 6$ : (a) $R a=1 \times 10^{6}$, (b) $R a=2 \times 10^{5}$.

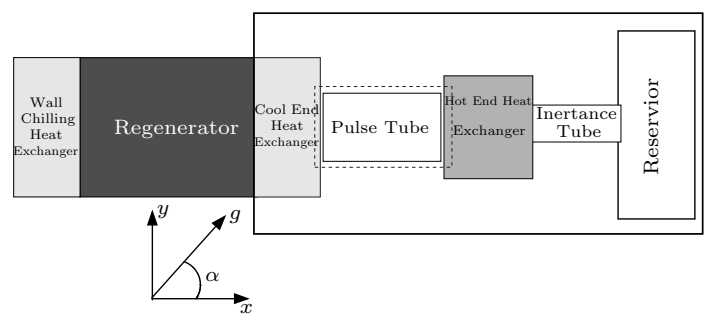

Fig. 6. The pulse tube refrigerator model.

In the temperature distribution of these two figures, we find that the temperature with no taper is chaotic, while the distribution with a taper is similar to the case of pure heat conduction. This fact once again proves that the flow intensity is suppressed in the cavity with a taper. The role the taper plays is to suppress the fluid flow and make the flow aspire to pure heat conduction.

Next we compare the maximum velocity and streaming function in different $\xi$ with inclination angle $\alpha=180^{\circ}$ and the temperature difference $\Delta T=200 \mathrm{~K}$, as shown in Fig. 4. We find that the values of the maximum velocity and streaming function in $\xi=1$ are maximal compared with other $\xi$. Point $\xi=1$ is an extreme value. The suppression effects are more obvious when the $\xi$ is smaller for the interval $\xi=0 \sim 1$, and the suppression effects are more obvious when the $\xi$ is larger for the interval $\xi>1$. This implies that the length difference $L_{\text {coolwall }}-L_{\text {hotwall }}$ is larger, and the suppression effects are more considerable.

Then we set the temperature in the hot wall $T=$ $300 \mathrm{~K}$ and in the cool wall $T=200 \mathrm{~K}$ and $280 \mathrm{~K}$ to produce $\Delta T=100 \mathrm{~K}, R a=1 \times 10^{6}$ and $\Delta T=20 \mathrm{~K}$, where $R a=2 \times 10^{5}$ is employed to investigate if the taper can suppress natural convection in different $R a$ numbers. Here taper $\xi=1 / 6$ is chosen. The maximum streaming functions for different $R a$ are shown in Fig. 5. We find that the value of the streaming function with a taper is smaller than the value without taper in different $R a$. We also see that the taper can suppress the natural convection in $R a=2 \times 10^{6}$, $R a=1 \times 10^{6}$ and $R a=2 \times 10^{5}$. Thus, we can say that the taper can suppress the natural convection universally. The above results are pure natural convection without oscillating flow. In the following, we introduce this method into the oscillating flow of the thermoacoustic system and check if the taper pulse shape can suppress the natural convection.

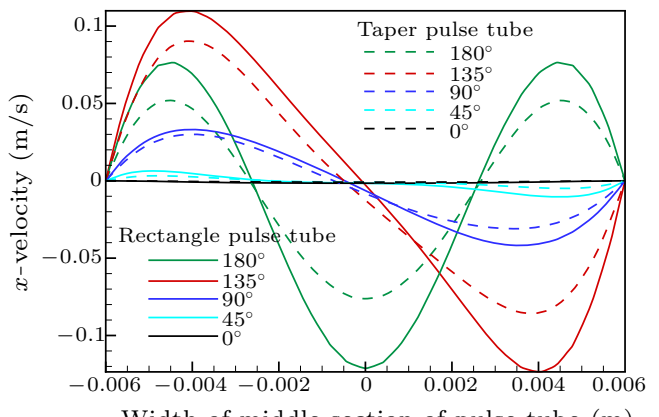

Width of middle section of pulse tube $(\mathrm{m})$

Fig. 7. Section velocity of the time-average oscillating flow in different pulse tube shapes.

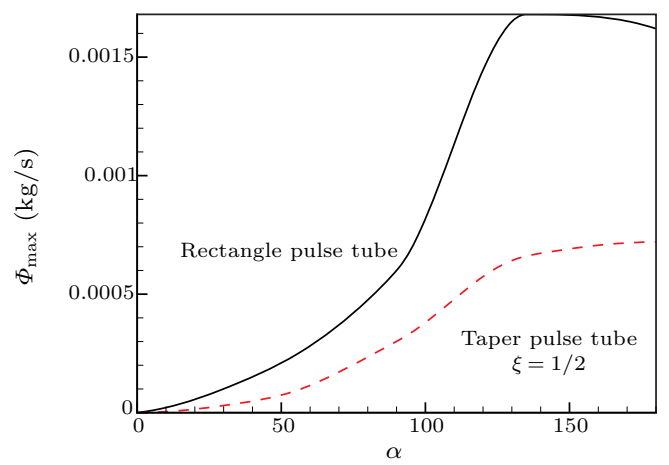

Fig. 8. Maximum streaming function distribution in different pulse tube shapes. 
Figure 6 is a pulse tube refrigerator model. The solid-line rectangular box is the simulation model for oscillating flow, which includes a cool-end heat exchanger, pulse tube, hot-end heat exchanger, inertia tube and reservoir.

For the oscillating flow model, the left wall of the cool-end heat exchanger adopts a pressure inlet boundary condition. Other walls are imposed under wall boundary conditions. The inflation pressure is under the condition of atmospheric pressure. Here we consider that the temperature of the inlet air sustains the temperature of the cool-end heat exchanger $100 \mathrm{~K}$. The walls of the hot-end heat exchanger, reservoir and inertance tube are isothermal walls with a temperature of $300 \mathrm{~K}$. The wall of the pulse tube is adiabatic. The initiation of the pulse tube is proposed to be a linear temperature distribution. Simulations continue until a steady periodic state is obtained. The criterion for steady periodic conditions is that the cycle-average temperature of the cold-end heat exchanger will reach a steady state. Here we choose the taper $\xi=1 / 2$ to investigate our problem. This cycle-averaged flow field is based on the average mass transport velocity values in the enclosure. The average mass transport (streaming) velocity is given by

$$
U_{\mathrm{st}}=\frac{\langle\rho u\rangle}{\langle\rho\rangle}, \quad V_{\mathrm{st}}=\frac{\langle\rho v\rangle}{\langle\rho\rangle},
$$

where \langle\rangle indicates the cycle-averaged quantities. ${ }^{[16]}$

Figure 7 is the distribution of the time-averaged velocity of the oscillating flow in the middle section of different pulse tubes. The abscissa is the width of the pulse tube. The vertical coordinate is the velocity distribution. From this figure, we find that the variation trend of the time-averaged velocity of the oscillating flow in different pulse tubes for every inclination is in general accord. The velocity magnitude increases gradually for both oscillating flows in two different pulse tube shapes when the inclination exceeds $90^{\circ}$. Comparing these two oscillating flows results, we can find that the time-averaged oscillating flow velocity in a rectangular pulse tube is larger than the time-averaged oscillating flow velocity in a taper pulse tube. Thus, the taper pulse tube shape has the effect of suppressing the natural convection. Figure 8 shows the distribution of the maximum streaming function in these two different pulse tubes. We can find that when the pulse tube has a taper, the streaming function obviously becomes smaller. The taper pulse tube decreases the natural convection.

In conclusion, previous scientists adopted a filling silk screen to decrease the gravity effect. However, these methods were unsatisfactory. In our research, we find that the cavity shape only changes from rectangular to taper, while the other conditions stay the same, and the natural convection is amazingly suppressed. We then introduce this idea to thermoacoustic engines and obtain satisfactory results for suppressing natural convection.

\section{References}

[1] Ostrach S 1988 J. Heat Transfer 1101175

[2] Khanafer K, Vafai K and Lightstone M 2003 Int. J. Heat Mass Transfer 463639

[3] Lee D Y, Park S J and Ro S T 1995 Int. J. Heat Mass Transfer $\mathbf{3 8} 2529$

[4] Thummes G et al 1997 Cryocoolers (New York: Springer) vol 9

[5] Trollier $\mathrm{T}$ et al 2004 Advances in Cryogenic Engineering vol 49 (New York: Springer)

[6] Ross R G and Johnson D L 2004 Cryogenics 44403

[7] Fujimoto S, Kang Y M and Matsubara Y 2000 Cryocoolers (New York: Springer) vol 10

[8] Johnson D L et al 1997 Cryocoolers (New York: Springer) vol 9

[9] Kasthurirengan S et al 2004 Advances in Cryogenic Engineering (New York: Springer) vol 49

[10] Shiraishi M et al 2004 Cryogenics 44101

[11] Shiraishi M, Fujisawa Y, Murakami M and Nanako A 2004 AIP Conf. Proc. $\mathbf{7 1 0} 1560$

[12] Patankar S V 1981 Numer. Heat Transfer Part A 4409

[13] Kalabin E V, Kanashina M V and Zubkov P T 2005 Numer. Heat Transfer Part A $4 \mathbf{7} 621$

[14] Kasthurirengan S, Thummes G and Heiden C 2000 Advances in Cryogenic Engineering (New York: Springer) vol 45

[15] Cha J et al 2006 Cryogenics 46658

[16] Aktas M K and Ozgumus T 2010 Int. J. Heat Mass Transfer $\mathbf{5 3} 5289$ 